\title{
Genome wide association analysis of the QTL MAS 2012 data investigating pleiotropy
}

\author{
Christine Grosse-Brinkhaus*, Sarah Bergfelder, Ernst Tholen \\ From 16th QTL-MAS Workshop \\ Alghero, Italy. 24-25 May 2012
}

\begin{abstract}
Background: Different genome wide association methods (GWAS) including multivariate analysis techniques were applied to identify quantitative trait loci (QTL) and pleiotropy in the simulated data set provided by the QTL-MAS workshop 2012 held in Alghero (Italy).

Methods: Genetic correlations and heritabilities for all three quantitative traits were obtained by a multivariate animal model. In a second step the data were corrected for a polygenic component containing the genomicbased kinship matrix. Residuals from this model were later used for QTL detection in a regression analysis, to achieve genome-wide rapid association (GRAMMAR). In order to take pleiotropic effects into account, all three traits were condensed via principle component techniques to two principal components (PC) which reflect the phenotypic variance covariance structure of all traits. The PCs were analyzed by single trait analysis by GRAMMAR. As an alternative to GRAMMAR, the data set was analyzed by Bayesian methods implemented in the package snptest. The program allows the analysis of the data in a univariate and a multivariate way, where all three traits are investigated simultaneously.
\end{abstract}

Results: According to the polygenic model, analyses the three traits revealed high heritability $(0.56,0.55$, and 0.66$)$. Traits 1 and 2 were highly correlated $\left(r_{g}=0.84\right)$. All applied GWAS revealed 10 QTL on four different chromosomes. No QTL was detected on chromosome 5. The Bayesian multivariate analysis revealed significant pleiotropic SNPS.

Conclusions: Principal component and multivariate analyses seem to be promising in order to characterize the genetic basis of trait relationships.

\section{Background}

Recently, the high-density single nucleotide polymorphism (SNP) arrays have been developed for almost all domestic animals. These tools offer the prerequisite of genome-wide association studies (GWAS), a powerful approach for high-resolution mapping of loci controlling phenotypic traits [1]. In agriculture many economically important traits share a common genetic background leading to positive or negative correlations $[2,3]$. Considering correlation effects (pleiotropy) in genomic selection allow to increase the mapping accuracy and to develop strategies to control unfavourable effects on a correlated trait.

\footnotetext{
* Correspondence: cgro@itw.uni-bonn.de

Institute of Animal Science, Group of Animal Breeding and Husbandry, University of Bonn, Endenicher Allee 15, 53115 Bonn, Germany
}

The aim of this study was to apply different genome wide association methods to identify quantitative trait loci (QTL) in the simulated data set provided by the QTL-MAS workshop 2012 Alghero (Italy) and to investigate pleiotropic effects among the three simulated quantitative traits.

\section{Methods}

In a first step genetic correlations and heritabilities for all three quantitative traits were obtained by a multivariate animal model analyzed by VCE6 [4]. In order to condensate the 3 traits, principal component techniques were applied based on the phenotypic correlation matrix. Resulting principal components (PCs) were used as additional phenotypes for GWAS. 
A quality control was performed for 10000 SNPs equally spaced on five chromosomes using a minor allele frequency $<0.01$ and a significant deviation from Hardy Weinberg equilibrium $(\mathrm{p}<0.01)$. Markers, which deviated from these criteria, were removed from the data set, so that 9596 were used for the GWAS.

The GWAS was performed with the genome-wide rapid association using mixed model and regression (GRAMMAR) [5]. Residuals were calculated for all traits by means of a polygenic model containing the genomicbased kinship matrix:

$$
\mathbf{y}_{\mathbf{i}}=\mu+a_{i}+e_{i}
$$

where $\mathbf{y}_{\mathbf{i}}$ is the phenotype (trait or principal component) of the $i^{\text {th }}$ individual, $\boldsymbol{a}_{\boldsymbol{i}}$ are the random additive polygenic effects with $a \sim N\left(0, \mathrm{G} \sigma_{a}^{2}\right)$

and $\mathbf{e}_{i}$ are the random residual effects. The kinship coefficients $\mathbf{G}$ from genomic data were estimated using the formula [6]:

$$
G_{i j}=\frac{1}{n} \sum_{k=1}^{n} \frac{\left(g_{i k}-p_{k}\right)\left(g_{j k}-p_{k}\right)}{p_{k}\left(1-p_{k}\right)}
$$

where $g_{i k}$ is the genotype of the $i^{t h}$ person at the $k^{\text {th }}$ SNP, $p_{k}$ is the frequency of the major allele and $n$ is the number of SNPs used for kinship estimation.

The residuals were estimated as

$$
y_{i} *=y_{i}-\left(\hat{\mu}+\hat{a}_{i}\right)
$$

The test for association was performed with these residuals using a linear model:

$$
\gamma_{i} *=\mu *+k g_{i}+e_{i} *
$$

where $y^{*}$ represents residuals from model (3) of $i^{t h}$ individuals (), $\mu^{*}$ the intercept, $k$ is the regression on the genotype $\left(\boldsymbol{g}_{\boldsymbol{i}}\right)$, where $\mathbf{g}$ contains a dose effect of a target allele for each SNP and $e^{*}$ is the random residual [7]. A $\mathrm{X}^{2}$ test-statistic is used to determine whether a SNP is significantly associated with the trait.

In addition permutation resampling techniques, as implemented in GenABEL [8], were used to correct for multiple testing. Genome wide significance (P-value < $0.05)$ was derived by applying 1000 permutations.

As an alternative to GRAMMAR, the data set was analyzed with Bayesian methods implemented in the package snptest [9]. The program allows the analysis of data in a univariate and a multivariate way, where all 3 traits are investigated simultaneously. The following Bayesian multivariate model was applied on the three traits:

$$
\left(\gamma_{i 1}^{*}, \ldots, \gamma_{i q}^{*}\right)^{T}=C\left(\beta_{1}, \ldots, \beta_{q}\right)^{T}+\left(e_{i 1}, \ldots, e_{i q}\right)^{T} \text { where }\left(e_{i 1}, \ldots, e_{i q}\right)^{T} \sim N_{q}(0, \Sigma)
$$

with $\left(y_{i 1}^{*}, \ldots, y_{i q}^{*}\right)$ is the vector of the residuals from model (3) measured on the $i^{\text {th }}$ individual. The residuals of the three traits $(q)$ were scaled to a mean of zero and a unit variance. $\mathbf{C}_{i}$ is the coded version of the genotype of the $i^{\text {th }}$ individual. For this model a conjugated prior was used that based on an inverse Wishart prior IW(c, Q) on the error covariance matrix $\Sigma$ and a matrix normal $(\mathbf{N})$ prior on the vector of parameters:

$$
\left(\beta_{1}, \ldots, \beta_{q}\right)-M \sim N(V, \Sigma),
$$

where $\mathbf{M}$ is a mean vector and $\mathrm{V}$ is a constant. Further information of the matrix nomal distribution can be found in Dawid [10]. For the priors the default values (IW(6,4Iqxq), $\mathbf{M}=0, \mathrm{~V}=0.02$ ) were used as recommended by the authors Marchini and Howie [9].

The Bayes Factor (BF) is the ratio of marginal likelihoods between a model of association $\left(M_{1}\right)$ and a null model $\left(\mathrm{M}_{0}\right)$ of no association:

$$
B F=\frac{P\left(\text { Data } \mid M_{1}\right)}{P\left(\text { Data } \mid M_{0}\right)}
$$

\section{Results and discussion \\ Polygenic investigation}

According to the polygenic model analysis the three traits revealed high heritability $(0.56,0.55$, and 0.66$)$. As a result of step 1, particular traits 1 and 2 were highly correlated $\left(r_{g}=0.84\right)$ (Table 1). A strong genetic correlation among trait 1 and trait 2, a negative correlation between trait 1 and trait 3 and a low positive correlation between trait 2 and trait 3 were observed. In order to investigate pleiotropy, all three traits were rearranged via principal component techniques to 3 independent principal components $(\mathrm{PC})$. The variances explained by each PC were $62.1 \%, 37.5 \%$ and $0.4 \%$, respectively (Table 2). PC 3 was excluded for further analyses, because of the low variance explained. PC1 was signifi-

\begin{tabular}{|c|c|c|c|}
\hline$h^{2}$ & Trait 1 & Trait 2 & Trait 3 \\
\hline Trait 1 & $0.56( \pm 0.04)$ & $0.84( \pm 0.02)$ & $-0.43( \pm 0.06)$ \\
\hline Trait 2 & 0.82 & $0.55( \pm 0.04)$ & $0.11( \pm 0.07)$ \\
\hline Trait 3 & -0.44 & 0.14 & $0.66( \pm 0.03)$ \\
\hline
\end{tabular}
cantly correlated with trait 1 and trait 2 whereas PC2 was mainly influenced by the relationship between trait 2 and trait 3 (Table 2).

\section{Single trait analysis using GRAMMAR}

Applying the GRAMMAR approach and correcting for multiple testing six, nine and 11 significant (genome

Genetic correlations and standard. errors above the diagonal. Phenotypic correlations below the diagonal. 
Table 2 Canonical correlation coefficients and proportions of the variance explained by each principal component (PC).

\begin{tabular}{ccccc}
\hline & Trait 1 & Trait 2 & Trait 3 & \% of variance \\
\hline PC 1 & 0.99 & 0.86 & -0.36 & 62.1 \\
PC 2 & -0.09 & 0.50 & 0.93 & 37.5 \\
PC 3 & 0.07 & -0.07 & 0.04 & 0.4 \\
\hline
\end{tabular}

wide P-value $<0.05)$ SNPs for trait 1, 2 and 3, respectively (Figure 1, Table 3) were identified. These SNPs comprised 10 QTL on 4 different chromosomes, where a QTL region was defined by using a $10 \mathrm{Mb}$ interval around the significant SNPs. Nominal P-values of these SNPs were close to zero (7E-06 - 2E-24). Regarding simulated true QTL in the QTL-MAS 2012 data set [11] no false positive QTL were detected. As described in methods, nominal P-values were corrected by permutation techniques in order to avoid false positives. However, this test might be too conservative. As Johnson et al. [12] described, if the correction for multiple testing is overly conservative or power is inadequate the risk of false negatives (Type 2 errors) increases. This might serve as an explanation that several QTL with small effects were missed in our analysis.

\section{Multivariate analysis}

Additionally, the two identified principal components (PC1, PC2) were treated as independent phenotypes analyzed with GRAMMAR and allowed to investigate pleiotropy between the traits. For PC1 three and for PC2 seven QTL regions were identified (Table 3). Principal components are uncorrelated and reflect the phenotypic variance covariance structure of traits. This might be helpful for genomic selection when negatively correlated traits are processed. Furthermore, several authors described that the analysis of PCs were generally more powerful and accurate than the single trait analysis $[13,14]$. Although a higher statistical power can be
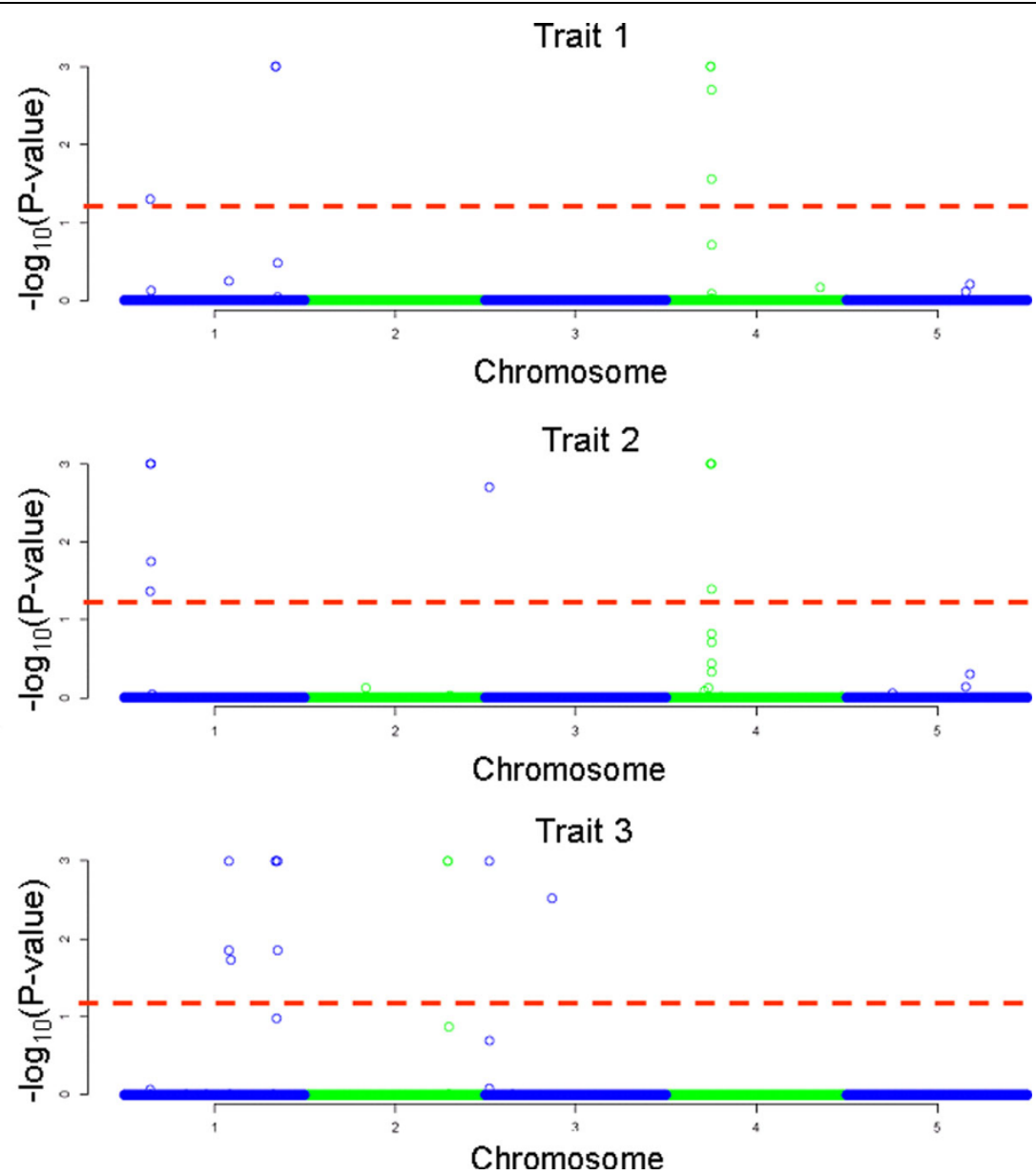

Figure 1 Manhattan plot for the three traits. One thousand permutations were used to identify genome wide significant thresholds. The dotted lines mark a genome-wide significance level of empirical p-value 0.05 . 
Table 3 Identified significant SNP using GRAMMAR approach.

\begin{tabular}{|c|c|c|c|c|c|}
\hline trait & chr. & position $^{a}$ & effect $^{b}$ & $s e^{c}$ & $X^{2 d}$ \\
\hline \multirow[t]{6}{*}{ Trait 1} & 1 & 84.05 & 14.81 & 3.45 & $18.39^{* * *}$ \\
\hline & 1 & 84.10 & -13.97 & 3.48 & $16.09^{* * *}$ \\
\hline & 4 & 24.85 & -14.74 & 3.84 & $14.70^{* * *}$ \\
\hline & 4 & 24.90 & 23.06 & 3.47 & $44.23^{* * *}$ \\
\hline & 4 & 25.00 & 12.64 & 3.40 & $13.83^{* *}$ \\
\hline & 4 & 25.25 & 13.58 & 3.97 & $11.69^{*}$ \\
\hline \multirow[t]{9}{*}{ Trait 2} & 1 & 14.60 & -0.96 & 0.19 & $26.20^{* * *}$ \\
\hline & 1 & 14.70 & 0.62 & 0.18 & $11.80^{*}$ \\
\hline & 1 & 14.75 & 0.65 & 0.18 & $12.61^{*}$ \\
\hline & 1 & 14.85 & 0.87 & 0.22 & $16.28^{* * *}$ \\
\hline & 3 & 2.15 & -1.04 & 0.27 & $14.39^{* *}$ \\
\hline & 4 & 24.85 & -0.83 & 0.21 & $16.37^{* * *}$ \\
\hline & 4 & 24.90 & 1.40 & 0.19 & $57.50^{* * *}$ \\
\hline & 4 & 25.00 & 0.77 & 0.18 & $17.93^{* * *}$ \\
\hline & 4 & 25.25 & 0.73 & 0.21 & $11.83^{*}$ \\
\hline \multirow[t]{11}{*}{ Trait 3} & 1 & 58.00 & -0.0021 & 0.0006 & $13.17^{* * *}$ \\
\hline & 1 & 58.25 & -0.0018 & 0.0005 & $10.96^{*}$ \\
\hline & 1 & 58.85 & 0.0013 & 0.0004 & $10.61^{*}$ \\
\hline & 1 & 84.05 & -0.0025 & 0.0004 & $39.27^{* * *}$ \\
\hline & 1 & 84.10 & 0.0024 & 0.0004 & $37.30^{* * *}$ \\
\hline & 1 & 84.80 & 0.0017 & 0.0005 & $10.93^{*}$ \\
\hline & 1 & 84.90 & -0.0019 & 0.0004 & $23.59^{* * *}$ \\
\hline & 2 & 79.15 & -0.0015 & 0.0004 & $14.41^{* * *}$ \\
\hline & 2 & 79.20 & -0.0023 & 0.0004 & $29.32^{* * *}$ \\
\hline & 3 & 2.15 & -0.0022 & 0.0006 & $14.26^{* * *}$ \\
\hline & 3 & 36.85 & -0.0014 & 0.0004 & $12.50^{* *}$ \\
\hline \multirow[t]{7}{*}{ PC 1} & 1 & 14.60 & -0.07 & 0.02 & $12.98^{*}$ \\
\hline & 1 & 84.05 & 0.07 & 0.02 & $14.30^{* *}$ \\
\hline & 1 & 84.10 & -0.07 & 0.02 & $12.23^{*}$ \\
\hline & 4 & 24.85 & -0.09 & 0.02 & $16.08^{* * *}$ \\
\hline & 4 & 24.90 & 0.14 & 0.02 & $49.33^{* * *}$ \\
\hline & 4 & 25.00 & 0.07 & 0.02 & $15.27^{* *}$ \\
\hline & 4 & 25.25 & 0.08 & 0.02 & $12.42^{*}$ \\
\hline \multirow[t]{10}{*}{ PC 2} & 1 & 14.60 & -0.07 & 0.02 & $15.50^{* * *}$ \\
\hline & 1 & 14.70 & 0.05 & 0.02 & $10.35^{*}$ \\
\hline & 1 & 84.05 & -0.09 & 0.02 & $30.50^{* * *}$ \\
\hline & 1 & 84.10 & 0.09 & 0.02 & $29.76^{* * *}$ \\
\hline & 1 & 84.90 & -0.07 & 0.02 & $19.80^{* * *}$ \\
\hline & 2 & 79.15 & -0.07 & 0.02 & $16.33^{* * *}$ \\
\hline & 2 & 79.20 & -0.09 & 0.02 & $26.57^{* * *}$ \\
\hline & 3 & 2.15 & -0.11 & 0.02 & $21.32^{* * *}$ \\
\hline & 3 & 2.30 & -0.08 & 0.02 & $10.40^{*}$ \\
\hline & 3 & 36.85 & -0.06 & 0.02 & $10.57^{*}$ \\
\hline
\end{tabular}

a: position in $\mathrm{Mb}$, b: additive effect of the trait, $\mathrm{c}$ : standard error of the additive effect, $d$ : chi-squared value with genome wide $p$-value applying 1000 permutations (***: $\left.\mathrm{P}<0.001 ;{ }^{* *}: \mathrm{P}<0.01,{ }^{*}:<0.05\right), \mathrm{PC}$ : principal component

achieved by this approach, a clear biological interpretation is hardly possible. Moreover, only pleiotropic QTL creating correlations between traits in the direction of
Table 4 Identified significant SNP using a multivariate Bayesian analysis method.

\begin{tabular}{ccc}
\hline Chr. & Position & Bayes factor \\
\hline 1 & 14.60 & 4.3952 \\
& 84.05 & 8.0932 \\
& 84.10 & 7.6726 \\
& 84.90 & 3.9167 \\
\hline 2 & 79.15 & 3.8483 \\
& 79.20 & 7.2374 \\
\hline 3 & 2.15 & 4.5439 \\
\hline 4 & 24.90 & 10.283 \\
\hline
\end{tabular}

Significant levels were obtained as described by Kass and Raftery [11].

phenotypic and/or genetic correlations can be detected with this approach $[13,15]$.

The Bayesian multivariate analysis revealed significant SNPs only involved in pleiotropy for all traits (Table 4). In total six QTL were detected using the significance and suggestive level described by Kass and Raftery [16] (Figure 2). Xu et al. [17] reviewed several publications and summarized them according to the advantages of Bayesian multivariate QTL analysis. A multivariate QTL analysis would increase the power and the precision of the pleiotropic QTL position, because the correlation structure of the investigated traits is considered $[17,18]$. Multivariate analysis is especially beneficial when one of the traits has a low heritability [19].

The effects of the SNPs on the genetic correlations were evaluated including the SNPs identified for the particular trait or PC (Table 3 Table 4) as fixed effect within the animal model, equation (1). The estimates of the genetic correlations between the particular traits are listed in Table 5. The genetic correlations were not necessarily reduced considering putative pleiotropic SNPs especially when the SNPs were not involved in the genetic architecture of the particular trait e.g. the analysis of PC1 for trait 1 and trait 3.

\section{Conclusions}

The investigation of the QTL-MAS 2012 data set using different multivariate approaches allowed identifying most of the simulated QTL with large effects. Smaller effects might not be detected due to the chosen threshold correction. The analysis of the PCs and multivariate approaches seem to be promising in order to detect QTLs mainly involved in pleiotropic effects.

Competing interests

The authors declare that they have no competing interests.

Authors' contributions

CG compiled the data set and performed the GRAMMAR and multivariate analyses. SB estimated the genetic parameters. CG and SB wrote the 


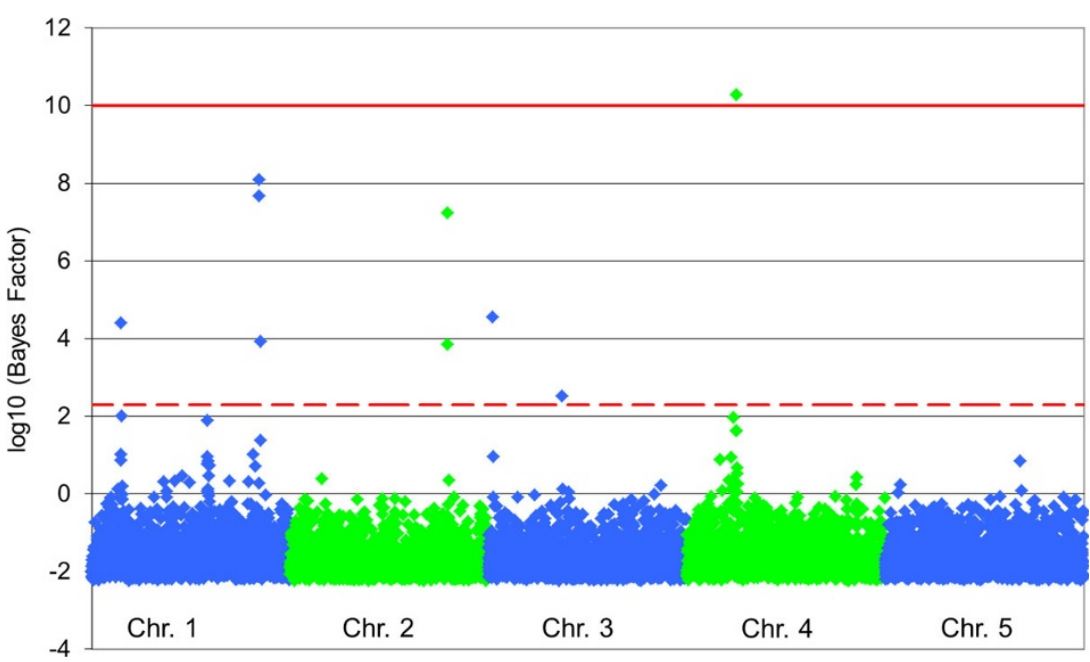

Figure 2 Manhattan plot of the Bayesian multivariate analysis. The red lines mark a significant (solid line) and a suggestive (dash line) level as described by Kass and Raftery [11].

\section{Table 5 Genetic correlations with and without fitting identified SNPs with the different association analyses as fixed effects.}

\begin{tabular}{lccc}
\hline & trait 1/trait 2 & trait 1/trait 3 & trait 2/trait 3 \\
\hline Single trait analysis* & 0.79 & -0.36 & 0.08 \\
PC1 & 0.81 & -0.46 & 0.13 \\
PC2 & 0.86 & -0.45 & 0.03 \\
Bayesian multivariate & 0.81 & -0.46 & 0.08
\end{tabular}

* Only significant associated SNPs of the particular traits were implemented as fixed effects in the polygenic model.

manuscript. ET advised analyses, data interpretation and revised the manuscript.

\section{Acknowledgements}

We thank the anonymous reviewer for the careful reading of our manuscript and the valuable comments. Sarah Bergfelder was supported by the European Union and Ministry for Economic Affairs, Energy and Industry of the Federal State of North Rhine-Westphalia (Grant no. 005-NA02-018C, project pigGS)

\section{Declarations}

The publication was funded by the University of Bonn, Institute of Animal Science, Germany.

This article has been published as part of BMC Proceedings Volume 8 Supplement 5, 2014: Proceedings of the 16th European Workshop on QTL Mapping and Marker Assisted Selection (QTL-MAS). The full contents of the supplement are available online at http://www.biomedcentral.com/bmcproc/ supplements/8/S5

Published: 7 October 2014

\section{References}

1. Andersson L: Genome-wide association analysis in domestic animals: a powerful approach for genetic dissection of trait loci. Genetica 2009, 136:341-349.

2. Cheverud JM: The genetic architecture of pleiotropic relations and differential epistasis. San Diego CA: Academic Press; 2001.

3. Falconer DS, Mackay TFC: Introduction to quantitative genetics. Harlow, England; New York: Prentice Hall; 41996.
4. Groenefeld E, Kovac M, Mielenz N: VCE User's Guide and Reference Manual Version 6.0. 2010.

5. Aulchenko YS, de Koning DJ, Haley C: Genomewide rapid association using mixed model and regression: A fast and simple method for genomewide pedigree-based quantitative trait loci association analysis. Genetics 2007, 177:577-585.

6. Astle W, Balding DJ: Population Structure and Cryptic Relatedness in Genetic Association Studies. Stat Sci 2009, 24:451-471.

7. Amin N, van Duijn CM, Aulchenko YS: A Genomic Background Based Method for Association Analysis in Related Individuals. PLoS One 2007, 2.

8. Aulchenko YS, Ripke S, Isaacs A, Van Duijn CM: GenABEL: an R library for genorne-wide association analysis. Bioinformatics 2007, 23:1294-1296.

9. Marchini J, Howie B: Genotype imputation for genome-wide association studies. Nat Rev Genet 2010, 11:499-511.

10. Dawid AP: Some Matrix-Variate Distribution-Theory - Notational Considerations and a Bayesian Application. Biometrika 1981, 68:265-274.

11. Usai MG: Simulated data and Comparative analysis of submitted results on QTL mapping and applied methods. 16th QTLMAS workshop 24-25 May, Alghero, Italy 2012 [http://qtl-mas-2012.kassiopeagroup.com/en/ program.php].

12. Johnson RC, Nelson GW, Troyer JL, Lautenberger JA, Kessing BD, Winkler CA, O'Brien SJ: Accounting for multiple comparisons in a genome-wide association study (GWAS). BMC Genomics 2010, 11.

13. Gilbert H, Le Roy P: Methods for the detection of multiple linked QTL applied to a mixture of full and half sib families. Genet Sel Evol 2007, 39:139-158.

14. Klei L, Luca D, Devlin B, Roeder K: Pleiotropy and principal components of heritability combine to increase power for association analysis. Genet Epidemiol 2008, 32:9-19.

15. Mangin B, Thoquet P, Olivier J, Grimsley NH: Temporal and multiple quantitative trait loci analyses of resistance to bacterial wilt in tomato permit the resolution of linked loci. Genetics 1999, 151:1165-1172.

16. Kass RE, Raftery AE: Bayes Factors. J Am Stat Assoc 1995, 90:773-795.

17. Xu CW, Wang XF, Li ZK, Xu SZ: Mapping QTL for multiple traits using Bayesian statistics. Genet Res 2009, 91:23-37.

18. Knott SA, Haley CS: Multitrait least squares for quantitative trait loci detection. Genetics 2000, 156:899-911.

19. Sorensen P, Lund MS, Guldbrandtsen B, Jensen J, Sorensen D: A comparison of bivariate and univariate QTL mapping in livestock populations. Genet Sel Evol 2003, 35:605-622.

doi:10.1186/1753-6561-8-S5-S2

Cite this article as: Grosse-Brinkhaus et al:: Genome wide association analysis of the QTL MAS 2012 data investigating pleiotropy. BMC Proceedings 2014 8(Suppl 5):S2. 